\title{
Obstetrical Healthcare Personnel's Attitudes and Perceptions on Maternal Vaccination with Tetanus-Diphtheria-Acellular Pertussis and Influenza
}

\author{
Vini Vijayan, ${ }^{1}$ Matthew Kim, ${ }^{2}$ Kenneth M. Zangwill, ${ }^{3}$ Chrisanna Mink, ${ }^{3}$ and Sylvia Yeh ${ }^{3}$ \\ ${ }^{1}$ Division of Immunology, Rheumatology and Infectious Diseases, Department of Pediatrics, University of Florida, \\ 1600 SW Archer Road, P.O. Box 100296, Gainesville, FL 32610, USA \\ ${ }^{2}$ Division of Obstetrics and Maternal Fetal Medicine, Cedars-Sinai Medical Center, Los Angeles, CA 90048, USA \\ ${ }^{3}$ Los Angeles Biomedical Research Institute at Harbor-UCLA Medical Center, Torrance, CA 90502, USA \\ Correspondence should be addressed to Vini Vijayan; vvijayan@ufl.edu
}

Received 4 February 2013; Accepted 2 April 2013

Academic Editor: Sherri L. LaVela

Copyright (C) 2013 Vini Vijayan et al. This is an open access article distributed under the Creative Commons Attribution License, which permits unrestricted use, distribution, and reproduction in any medium, provided the original work is properly cited.

Objectives. To assess perceptions of obstetrical healthcare personnel (HCP) regarding routine delivery of Tdap and influenza vaccines to pregnant and postpartum women and identify perceived barriers to vaccination. Methods. Anonymous Web-based survey of obstetricians and nurses caring for pregnant and/or postpartum women. Results. We contacted $342 \mathrm{HCP}$ and received 163 (48\%) completed surveys (33/142 (23\%) obstetricians, 130/200 (65\%) nurses). Among obstetricians, $72 \%$ and $63 \%$ thought it was "beneficial" to immunize postpartum women against influenza and pertussis, respectively. Only $8 \%$ reported vaccinating $>75 \%$ of pregnant women in their care against influenza. Similarly, $<1 \%$ of obstetricians reported vaccinating against pertussis. Of all HCP surveyed, $92 \%$ and $58 \%$ were familiar with ACIP recommendations for influenza and pertussis, respectively. Reported perceived barriers included patient refusal to be vaccinated, reimbursement difficulties, and discomfort in providing vaccine education. Ninety-four percent of respondents agreed that standing orders would be helpful to ensure postpartum vaccination. Conclusions. HCP were less familiar with ACIP recommendations for Tdap compared to influenza vaccines. Substantial discrepancy existed between perceived benefit of vaccination and reported immunization practices. Most identified barriers could be addressed with provider training; however, other barriers require review and changes in systematic policies related to vaccine reimbursement.

\section{Introduction}

The Advisory Committee on Immunization Practices (ACIP) recommends influenza vaccination of pregnant or postpartum women as well as household contacts of infants of $<1$ year of age $[1,2]$. The ACIP also recommends tetanus toxoid, reduced diphtheria toxin, and acellular pertussis vaccine (Tdap) for postpartum women and household contacts of infants [1]. Despite these recommendations, only $11 \%$ of pregnant women and approximately $25 \%$ of household contacts of high risk individuals were vaccinated against influenza in 2008 [2]. Similarly, only 5\% of adult household contacts of an infant of $<1$ year of age received Tdap in 2008 [3]. Over the last 2 years, influenza vaccination coverage increased to
45\% for 2009 monovalent $\mathrm{H} 1 \mathrm{~N} 1$ vaccine in 2009 and this was sustained for seasonal influenza vaccination in 2010 [4-6], likely secondary to mass media attention during the 2009$2010 \mathrm{H} 1 \mathrm{N1}$ influenza pandemic.

These recommendations exist as pertussis and influenza are highly contagious vaccine-preventable illnesses to which infants of $<6$ months of old are at risk for significant morbidity and/or mortality compared to older ages. Because of their young age, these infants are incompletely immunized against pertussis and are not eligible for immunization against influenza, placing them at increased risk for infection [7-10]. Therefore, attention to vaccination opportunities in the postpartum period for mothers and household contacts of infants has developed $[1,2]$. 
TABLE 1: Characteristics of surveyed obstetricians and nurses to determine knowledge, attitudes, and perceived barriers to vaccination of pregnant and/or postpartum women ${ }^{1}$.

\begin{tabular}{lcc}
\hline & Obstetricians & Nurses \\
\hline $\begin{array}{l}\text { Duration of practice after } \\
\text { training }\end{array}$ & & \\
$\quad<5$ years & $14 / 33(42 \%)$ & $34 / 130(26 \%)$ \\
$\quad 5-\leq 10$ & $7 / 33(21 \%)$ & $32 / 130(25 \%)$ \\
$>10-\leq 20$ & $4 / 33(12 \%)$ & $30 / 130(23 \%)$ \\
$>20$ & $8 / 33(24 \%)$ & $34 / 130(26 \%)$ \\
Employment setting & & \\
$\quad$ Solo & $4 / 30(13 \%)$ & $0 / 130(0 \%)$ \\
$\quad$ Hospital based & $18 / 30(60 \%)$ & $129 / 130(99 \%)$ \\
$\quad$ Group practice & $8 / 30(26 \%)$ & $1 / 130(0.8 \%)$ \\
Number of pregnant women & & \\
seen per week & & \\
$\quad<5$ & $3 / 32(9 \%)$ & $41 / 130(32 \%)$ \\
$5-\leq 10$ & $5 / 32(15 \%)$ & $20 / 130(15 \%)$ \\
$>10-\leq 20$ & $6 / 32(19 \%)$ & $41 / 130(31 \%)$ \\
$>20-\geq 50$ & $12 / 32(37 \%)$ & $15 / 130(11 \%)$ \\
$>50$ & $6 / 32(19 \%)$ & $13 / 130(10 \%)$ \\
\hline
\end{tabular}

${ }^{1}$ Not all questions were answered by the entire group. Denominator shown represents number of responses per question in that group.

Attitudes and beliefs held by healthcare personnel (HCP) may impact the ability to successfully implement vaccination during pregnancy and the postpartum period [11, 12]. The objectives of this study were to assess attitudes, knowledge, and self-reported compliance of obstetrical HCP regarding delivery of Tdap and influenza vaccines and their perceptions-related vaccination of pregnant and postpartum women and their household contacts.

\section{Methods}

This study was conducted at Cedars-Sinai Medical Center (CSMC) located at Los Angeles, CA, USA, during July and August 2009 and approved by the CSMC institutional review board as well as our local institutional review board. CSMC has more than 6000 deliveries per year and serves a diverse ethnic population, of which $58 \%$ is White, $17 \%$ Hispanic, $14 \%$ Asian, 9\% Black, and 2\% others. CSMC serves patients of all socioeconomic backgrounds with many insurance carriers including Medi-Cal, the Medicaid program in California. During labor and delivery, obstetric patients are cared for by the CSMC seven-member hospital-based groups, obstetrical residents in training, and community private practitioners. Hospital-based physicians also provide routine prenatal care in outpatient clinics. At the time of the study, no defined hospital policies existed regarding influenza or pertussis vaccinations of postpartum mothers. Of note, the ACIP recommendations for postpartum Tdap administration were published in May 2008, over one year before this study was conducted.

We performed an anonymous Web-based survey, which was sent to all obstetricians with admitting privileges at CSMC (hospital and private practice based), obstetrical nurse practitioners, and nurses. These groups were recruited through an institutional email to complete the survey. This survey was adapted from a previously validated survey [12] and was designed to assess HCP knowledge of the ACIP recommendations related to Tdap and influenza vaccines as well as attitudes and practices of HCP related to vaccination of pregnant and postpartum women. We asked HCP to complete the following statements: "Having all postpartum women properly immunized against influenza is" (1) "useless," (2) "may be beneficial," or (3) "beneficial," and "Having all postpartum women properly immunized against pertussis is," (1) "useless," (2) "may be beneficial," or (3) "beneficial." The survey also inquired about several potential barriers to immunization. The questionnaire included 35-40 questions, depending on the group surveyed. The survey was pilot tested on a convenience sample of two obstetricians and three nurses in order to ensure clarity and ease of administration and refinements were made thereafter. The survey's readability was at an 11th grade level, as measured by the Flesch-Kincaid index. The respondents rated their answers to questions on a 3-point Likert scale, with zero equal to "not a barrier to immunization," (1) "maybe a factor," and (2) major barrier to immunization. The survey was distributed electronically using Survey Monkey (http://www.surveymonkey.com).

Utilizing the hospital Listserv, we sent an email which contained links to the survey website to the HCP involved in the care of pregnant or postpartum women. A reminder was sent weekly for three weeks, from July 2009 to August 2009. Respondent practice setting and length of time in that position were collected. After completion of the survey, respondents received a gift certificate for redemption at a local coffee shop.

2.1. Data Analysis. We generated descriptive analyses of the survey responses. The obstetricians and nurse responses were grouped separately unless otherwise specified. All of the comparisons were two tailed and a $P$ value of $\leq 0.05$ was considered statistically significant. For categorical variables, proportions were calculated and univariate analysis using Chi-squared or Fisher's exact test was performed as appropriate. All analyses were conducted using SAS version 9.2.

\section{Results}

We contacted 342 HCP and received 163 (48\%) completed surveys. This included 33/142 (23\%) obstetricians and 130/200 $(65 \%)$ nurses. No specific question was systematically left unanswered. As noted in Table 1, the majority of survey respondents were hospital-based HCP. Nineteen of thirtythree (58\%) obstetricians and 96/130 (74\%) nurses were in practice for $\geq 5$ years. The median number of patients seen was between 10 to 50 pregnant women per week. 
TABLE 2: HCP Knowledge of ACIP vaccination recommendations for pregnant and/or postpartum women and self-reported immunization practices.

\begin{tabular}{lc}
\hline Questions and answers & Obstetricians \\
\hline The ACIP recommends routine administration of Tdap to all postpartum women & \\
and their contacts. & $14 / 30(47 \%)$ \\
$\quad$ True & Nurses \\
The ACIP recommends administration of influenza vaccine to women who will be & $28 / 32(88 \%)$ \\
pregnant during the flu season. & \\
True & \\
What percent of pregnant women have you vaccinated against influenza in the past & $8 / 26(31 \%)$ \\
year? ${ }^{1,2}$ & $5 / 26(19 \%)$ \\
$0 \%-10 \%$ & $5 / 26(19 \%)$ \\
$\geq 10 \%-25 \%$ & $6 / 26(23 \%)$ \\
$\geq 25 \%-50 \%$ & $2 / 26(8 \%)$ \\
$\geq 50 \%-75 \%$ & $113 / 130(87 \%)$ \\
$\geq 75 \%-100 \%$ & $8 / 130(6 \%)$ \\
What percent of nonpregnant/postpartum women have you vaccinated against & $5 / 130(4 \%)$ \\
pertussis in past year? ${ }^{2}$ & $3 / 130(2 \%)$ \\
$0 \%-10 \%$ & $1 / 130(0.8 \%)$ \\
$\geq 10 \%-25 \%$ & $25 / 26(96 \%)$
\end{tabular}

${ }^{1} P \leq 0.05$, obstetricians versus nurses (logistic regression for increasing percentage by obstetricians).

${ }^{2}$ Not all questions were answered by the entire group. Denominator shown represents number of responses per question in that group.

3.1. Knowledge and Practices regarding Influenza and Tdap Vaccination. Regarding influenza, 23/32 (71\%) obstetricians and 64/123 (52\%) nurses thought it was "beneficial" to immunize postpartum women against influenza $(P>0.05)$. Of all the HCP surveyed, 92\% were aware that the ACIP recommended influenza vaccination in pregnancy (Table 2). Seventy percent $(21 / 30)$ of obstetricians reported that they routinely offered vaccines to pregnant women as part of their regular practice. However, only $2 / 26(8 \%)$ reported vaccinating $75 \%$ or more of pregnant women against influenza. Only $27 \%$ of obstetricians had "ever diagnosed" a patient with influenza. Of the nurses surveyed, $49 / 129$ (38\%) and 59/129 (46\%) answered "likely," and "very likely," respectively to the question, "How likely is it that you would support your postpartum patients to get vaccinated against influenza?"

For Tdap, 20/32 (62\%) of obstetricians and 59/123 (48\%) of nurses thought it was "beneficial" to immunize postpartum women against pertussis $(P>0.05)$. Of all the HCP surveyed, only $58 \%$ responded "true" to the statement, "The ACIP recommends routine administration of Tdap to all postpartum women and their household contacts." We found no statistical difference among obstetricians and nurses in their knowledge of the ACIP recommendations. Less than $1 \%$ of survey respondents reported vaccinating postpartum or nonpregnant women against pertussis in the past year. None of the obstetricians had diagnosed pertussis. Of the nurses surveyed, 54/129 (42\%) and 44/129 (34\%), respectively, answered "likely" and "very likely," respectively to the question, "How likely is it that you would support your postpartum patients to get vaccinated against pertussis?"
3.2. Barriers to Vaccination. Table 3 describes perceived barriers to the delivery of immunization to pregnant and postpartum women. Patient refusal to be vaccinated was the most commonly reported anticipated barrier among all survey respondents followed by issues related to reimbursement and cost. In addition, concerns regarding vaccine safety and lack of availability of the Tdap and influenza vaccines in their practice settings were also reported by $>75 \%$ of respondents. Seventy-seven percent of HCP perceived that legal liability "may be a factor" hindering vaccination of pregnant or postpartum women. In addition, a perceived lack of knowledge and therefore an ability to credibly educate patients regarding the risks and benefits of vaccination against influenza and pertussis were also considered a barrier to immunization. Nurses were more concerned about liability and counseling patients for Tdap vaccination compared to obstetricians $(P<$ $0.05)$.

Among the 21 obstetricians who routinely offered vaccines to women in their practices, the most important factors hindering actual vaccination were: (1) the patient had more urgent health problems and (2) time constraints. Liability was reported to be less of a concern in this group of obstetricians. They also reported that discomfort in providing education regarding vaccines was not a factor hindering vaccination. We found no significant differences in the responses between private versus hospital-based obstetricians.

We asked obstetricians "How likely is it that you would refer a patient to get vaccinated elsewhere?" Potential responses included "less likely," "likely," and "very likely." Sixty percent (12/20) of hospital-based obstetricians and 54\% (7/13) 
TABle 3: Perceived barriers to vaccination with Tdap and influenza vaccine among obstetricians and nurses.

\begin{tabular}{lcc}
\hline Perceived barriers to vaccination & \multicolumn{2}{c}{ Frequency in proportion ${ }^{2}(\%)$} \\
Patient refusal to vaccinate against pertussis & Obstetricians & $106 / 110(96 \%)$ \\
Reimbursement by insurance for Tdap & $29 / 31(94 \%)$ & $97 / 107(91 \%)$ \\
Cost of Tdap vaccine & $25 / 30(83 \%)$ & $83 / 105(79 \%)$ \\
Practice does not offer/stock Tdap vaccine & $24 / 31(77 \%)$ & $94 / 107(88 \%)$ \\
Uncomfortable in providing education, Tdap & $23 / 30(77 \%)$ & $74 / 107(69 \%)$ \\
Patient refusal to vaccinate against influenza & $16 / 30(53 \%)$ & $107 / 111(96 \%)$ \\
Reimbursement by insurance for influenza vaccine & $28 / 31(90 \%)$ & $99 / 110(90 \%)$ \\
Uncomfortable in providing education, influenza & $25 / 30(83 \%)$ & $74 / 109(68 \%)$ \\
Cost of influenza vaccine & $23 / 30(77 \%)$ & $83 / 110(75 \%)$ \\
Patient has more urgent health problems & $23 / 31(74 \%)$ & $85 / 109(78 \%)$ \\
Practice does not offer/stock influenza vaccine & $24 / 31(77 \%)$ & $87 / 108(81 \%)$ \\
Time constraints & $19 / 30(63 \%)$ & $75 / 109(69 \%)$ \\
Storage difficulty & $22 / 31(71 \%)$ & $60 / 108(56 \%)$ \\
Frequent change in immunization recommendations & $21 / 31(68 \%)$ & $82 / 108(76 \%)$ \\
Lack of personnel & $19 / 31(61 \%)$ & $62 / 110(56 \%)$ \\
Concerns regarding liability ${ }^{1}$ & $17 / 31(55 \%)$ & $92 / 109(84 \%)$ \\
\hline
\end{tabular}

${ }^{1} P \leq 0.05$, obstetricians versus nurses (Chi-squared test).

${ }^{2}$ Not all questions were answered by the entire group. Denominator shown represents number of responses per question in that group.

of the obstetricians in private practice reported that they were less likely to refer their patient elsewhere for the purpose of vaccination.

3.3. Attitudes to Postpartum Vaccination. We asked obstetricians which group of providers should bear responsibility for promoting vaccination in birth mothers with Tdap and influenza and more than one response was allowed. Forty-one percent (13/32) answered that the repsonsibility to vaccinate birth mothers was that of the obstetricians. Other groups that obstetricians felt had the responsibility to vaccinate postpartum women were the primary care provider $(22 \%)$, the pediatrician $(22 \%)$, the nurses $(25 \%)$, or all HCP $(65 \%)$. Twenty-eight percent (9/32) respondents chose more than one response.

Nearly $72 \%$ (23/32) of obstetricians and 81\% (100/123) of nurses considered postpartum immunization as "somewhat of a priority" or "high priority" as compared to other aspects of patient care. However, regardless of practice location and reported high levels of concern and barriers to vaccination, $95 \%$ of HCP ( $97 \%$ of obstetricians and $93 \%$ of nurses) agreed that use of standing orders for vaccination of postpartum women would ensure vaccination prior to discharge.

3.4. Barriers to Vaccination of Household Contacts. Among 31 obstetricians, only $5(16 \%)$ felt it was their responsibility to promote and administer influenza and Tdap vaccines to household contacts. They reported that other healthcare providers were responsible for this task including pediatricians (45\%), adult primary care physicians (26\%), nurses and hospital administration (26\%), or all of the above (48\%). Of note, $29 \%$ (9/31) of obstetricians chose more than one response.
We asked the HCP "Please indicate to what extent each of the following barriers hinders your interest or capacity to immunize household contacts of infants". Potential responses were, "not a factor", "maybe a factor", and "major factor" hindering immunization. In Table 4, significant barriers to vaccination of household contacts reported by both obstetricians and nurses are summarized. The major barriers to vaccination of household contacts were the perceived absence of a primary care physician to administer the vaccines and household contacts' potential lack of insurance. Concerns about immunizing household contacts in the hospital were legal liability and lack of awareness about immunization to prevent infections in the newborn among household contacts. A greater number of nurses than obstetricians reported these as major barriers compared to obstetricians $(P \leq 0.05)$.

\section{Discussion}

We believe our data are the first to assess the knowledge and perceptions of obstetrical HCP related to influenza and pertussis vaccination of pregnant and/or postpartum women. We found that a substantial majority of obstetricians reported familiarity with ACIP recommendations for vaccination of pregnant or postpartum women against influenza and considered it beneficial; however, the self-reported delivery of influenza vaccination to pregnant women varied greatly. HCP were less familiar with ACIP recommendations for Tdap. Factors that were most frequently reported by obstetricians to hinder vaccination were (1) anticipated patient refusal, (2) reimbursement for vaccination, (3) concern that the patient had more pressing healthcare needs, and (4) time constraints. For nurses and pharmacists, identified barriers 
TABLE 4: Obstetricians and nurses perceived barriers to vaccination of household contacts of infants with Tdap and influenza.

\begin{tabular}{lcc}
\hline \multirow{2}{*}{ Perceived barrier } & \multicolumn{2}{c}{ Major/maybe a barrier } \\
& Obstetricians & Nurses \\
\hline $\begin{array}{l}\text { Household contact has no } \\
\text { primary care physician }\end{array}$ & $26 / 30(86 \%)$ & $97 / 107(90 \%)$ \\
$\begin{array}{l}\text { Household contact has no } \\
\text { insurance }\end{array}$ & $22 / 30(73 \%)$ & $95 / 107(88 \%)$ \\
$\begin{array}{l}\text { HCP did not advise contact that } \\
\text { vaccine was needed }\end{array}$ & $25 / 30(83 \%)$ & $104 / 107(97 \%)$ \\
$\begin{array}{l}\text { Difficulty to get reimbursed } \\
\text { Concerns regarding liability }^{1}\end{array}$ & $23 / 30(76 \%)$ & $98 / 107(92 \%)$ \\
$\begin{array}{l}\text { Household contact does not } \\
\text { understand importance of }\end{array}$ & $22 / 30(73 \%)$ & $96 / 107(90 \%)$ \\
vaccination & $28 / 30(93 \%)$ & $102 / 107(95 \%)$ \\
\hline
\end{tabular}

${ }^{1} P \leq 0.05$, obstetricians versus nurses (Chi-squared test).

in vaccinating this population were similar to the additional concern regarding liability.

A high rate of HCP reported being uncomfortable providing risk and benefit education (53\%-77\%). Many strategies to address patient refusal to vaccinate exist which emphasize acknowledgement of patient fears followed by clear communication of supportable facts about vaccine effectiveness and safety. Repeatedly, a physician's recommendation has been demonstrated to improve patients' acceptance of vaccination [13-15]. Formally incorporating immunization education into routine obstetrical prenatal care may facilitate the likelihood of addressing maternal vaccine concerns, ensuring timeliness of vaccine uptake, and reduce nursing concerns of patient refusal. Time constraints, which have been reported as a barrier to vaccine risk-benefit communication in previous studies, were a concern for HCP in our study. This notion has been refuted by time-in-motion analysis studies in the pediatric setting, but data are not yet available for obstetrical settings [16].

The second most common barrier was concerns related to cost and reimbursement of vaccinations $[17,18]$. Currently, reimbursements for deliveries are usually based on a capitated system, without the ability to bill for additional services provided. This system may serve as a disincentive for participation in vaccine programs. Our study noted that hospitalbased obstetricians more frequently reported reimbursement concerns when compared to private practitioners although this finding was not statistically significant and we may have lacked power to detect a difference, if present. Directly addressing this issue, therefore, is essential for successful implementation of a vaccination program.

One notable finding of our survey was the fact that many obstetricians did not feel primarily responsible for the vaccination of their patients, but rather that vaccination after delivery should be a shared responsibility with primary physicians. In addition, they considered vaccination of household contacts to be the primary responsibility of pediatricians, although the age of household contact was not specified in the question. This uncertainty regarding who is responsible for discussing, recommending, and administering vaccines is consistent with prior studies regarding vaccines for obstetric patients [19-21]. The hesitancy related to vaccinating household contacts may be due to the lack of preexisting physicianpatient relationships and the lack of a sense of urgency or mandate (with associated legal protection) to act on the behalf of public health.

This study has several limitations. It was performed at a single metropolitan hospital which may not be generalizable to all obstetric practices. However, our findings are similar to other studies of adult practitioners with regard to vaccine knowledge and perceived barriers [18, 21, 22]. Our survey also relied on unaudited self-reporting of practices and vaccination rates, which may introduce bias. Since this study was conducted, the ACIP has made significant changes to their recommendations in an effort to reduce the burden of pertussis in infants. Currently, the ACIP recommends that Tdap be administered during every pregnancy, irrespective of the patient's prior history of receiving Tdap. If not administered during pregnancy, the vaccine was recommended to be administered postpartum. Although the perceived barriers to vaccination of pregnant and postpartum women may differ, most women identify their obstetrician as their source of primary care and may not be seen by another physician during their prenatal or postpartum period. Hence, the personal attitudes and beliefs held by obstetrical healthcare personnel may affect the likelihood that vaccination will be implemented during pregnancy and during the postpartum period.

Although influenza vaccination rates for pregnant women increased substantially in 2009-2010 and were sustained in 2010-2011 [4-6], postpartum vaccination prior to hospital discharge remains an important strategy for women who have not received these vaccines. A systems-based hospital postpartum immunization delivery strategy may provide the best opportunity to vaccinate women against Tdap and influenza to protect themselves and their infants. Encouraging patients to seek immunization in the hospital may also be a complementary strategy for increasing vaccination, especially if lack of a primary physician or insurance is an issue in obtaining vaccines during pregnancy [23-25].

Standing orders have been shown to be an effective method to increase vaccination rates in many vaccination outreach efforts $[26,27]$. Studies have also suggested that medical personnel such as nurses may be as or more effective at ensuring vaccination via standing orders than physicians $[27,28]$. Educational efforts for obstetricians and nurses including recommendations, rationale for vaccination, and vaccine risk-benefit communications training would be important in the success of a hospital-based postpartum vaccination program.

Our data begin to characterize the obstacles to vaccination of pregnant and postpartum women and household contacts of their newborn infants. Fortunately, there are well-researched and successful strategies available to address many of these barriers. We believe this information may be useful for hospitals and healthcare organizations to facilitate implementation of current recommendations for 
vaccination designed to provide protection against pertussis and influenza for pregnant women and newborn infants.

\section{Conflict of Interests}

The authors declare that they have no conflict of interest.

\section{Acknowledgment}

This paper was supported by Centers for Disease Control and Prevention, Atlanta, GA, USA, under Grant no. 5U01IP000192

\section{References}

[1] T. V. Murphy, B. A. Slade, K. R. Broder et al., "Prevention of pertussis, tetanus, and diphtheria among pregnant and postpartum women and their infants recommendations of the advisory committee on immunization practices (ACIP)," Morbidity and Mortality Weekly Report, vol. 57, no. 4, pp. 1-47, 2008.

[2] Centers for Disease Control and Prevention, "Prevention and control of influenza. Recommendations of the advisory committee on immunization practices (ACIP)," Morbidity and Mortality Weekly Report, pp. 1-60, 2008.

[3] Center for Disease Control and Prevention, "Tetanus and pertussis vaccination coverage among adults aged $>18$ yearsUnited States, 1999 and 2008," Morbidity and Mortality Weekly Report, vol. 59, no. 40, pp. 1302-1306, 2010.

[4] Centers for Disease Control and Prevention, "Interim results: influenza A (H1N1) 2009 monovalent vaccination coverageUnited States, October-December 2009," Morbidity and Mortality Weekly Report, vol. 59, pp. 44-48, 2010.

[5] Centers for Disease Control and Prevention, "Seasonal influenza and $2009 \mathrm{H} 1 \mathrm{~N} 1$ influenza vaccination coverage among pregnant women-10 States, 2009-10 influenza season," Morbidity and Mortality Weekly Report, vol. 59, pp. 1541-1545, 2010.

[6] H. Ding, T. A. Santibanez, D. J. Jamieson et al., "Influenza vaccination coverage among pregnant women-national 2009 H1N1 flu survey (NHFS)," The American Journal of Obstetrics and Gynecology, vol. 204, no. 6, supplement 1, pp. S96-S106, 2011.

[7] A. M. Wendelboe, E. Njamkepo, A. Bourillon et al., "Transmission of Bordetella pertussis to young infants," Pediatric Infectious Disease Journal, vol. 26, no. 4, pp. 293-299, 2007.

[8] E. A. Mortimer Jr., "Pertussis and its prevention: a family affair," Journal of Infectious Diseases, vol. 161, no. 3, pp. 473-479, 1990.

[9] E. L. Hewett and K. M. Edward, "Clinical practice: pertussis-not just for kids," The New England Journal of Medicine, vol. 352, pp. 1215-1222, 2005.

[10] K. A. Poehling, K. M. Edwards, G. A. Weinberg et al., "The underrecognized burden of influenza in young children," The New England Journal of Medicine, vol. 355, no. 1, pp. 31-40, 2006.

[11] K. L. Nichol and R. Zimmerman, "Generalist and subspecialist physicians' knowledge, attitudes, and practices regarding influenza and pneumococcal vaccinations for elderly and other high-risk patients," Archives of Internal Medicine, vol. 161, no. 22, pp. 2702-2708, 2001.

[12] R. Prislin, P. R. Nader, M. de Guire et al., "Physicians' immunization knowledge, attitudes, and practices. A valid and internally consistent measurement tool," The American Journal of Preventive Medicine, vol. 17, no. 2, pp. 151-152, 1999.

[13] R. C. Chi and K. M. Neuzil, "The association of sociodemographic factors and patient attitudes on influenza vaccination rates in older persons," The American Journal of the Medical Sciences, vol. 327, no. 3, pp. 113-117, 2004.

[14] R. Lyn-Cook, E. A. Halm, and J. P. Wisnivesky, "Determinants of adherence to influenza vaccination among inner-city adults with persistent asthma," Primary Care Respiratory Journal, vol. 16, no. 4, pp. 229-235, 2007.

[15] R. K. Zimmerman, T. A. Santibanez, J. E. Janosky et al., "What affects influenza vaccination rates among older patients? An analysis from inner-city, suburban, rural, and veterans affairs practices," The American Journal of Medicine, vol. 114, no. 1, pp. 31-38, 2003.

[16] P. G. Szilagyi, M. K. Iwane, S. E. Humiston et al., “Time spent by primary care practices on pediatric influenza vaccination visits: implications for universal influenza vaccination," Archives of Pediatrics and Adolescent Medicine, vol. 157, no. 2, pp. 191-195, 2003.

[17] A. R. Hinman, W. A. Orenstein, and L. Rodewald, "Financing immunizations in the United States," Clinical Infectious Diseases, vol. 38, no. 10, pp. 1440-1446, 2004.

[18] D. R. Johnson, K. L. Nichol, and K. Lipczynski, "Barriers to adult immunization," The American Journal of Medicine, vol. 121, no. 7, supplement 2, pp. S28-S35, 2008.

[19] M. L. Power, M. A. Leddy, B. L. Anderson, S. A. Gall, B. Gonik, and J. Schulkin, "Obstetrician-gynecologists' practices and perceived knowledge regarding immunization," The American Journal of Preventive Medicine, vol. 37, no. 3, pp. 231-234, 2009.

[20] S. J. Schrag, A. E. Fiore, B. Gonik et al., "Vaccination and perinatal infection prevention practices among obstetriciangynecologists," Obstetrics and Gynecology, vol. 101, no. 4, pp. 704-710, 2003.

[21] B. Gonik, T. Jones, and D. Contreras, "The obstetriciangynecologist's role in vaccine-preventable diseases and immunization," Morbidity and Mortality Weekly Report, vol. 54, no. 41, pp. 1050-1052, 2005.

[22] S. J. Clark, S. Adolphe, M. M. Davis, A. E. Cowan, and K. Kretsinger, "Attitudes of US obstetricians toward a combined tetanus-diphtheria-acellular pertussis vaccine for adults," Infectious Diseases in Obstetrics and Gynecology, vol. 2006, Article ID 87040, 5 pages, 2006.

[23] K. L. Nichol, "Ten-year durability and success of an organized program to increase influenza and pneumococcal vaccination rates among high-risk adults," The American Journal of Medicine, vol. 105, no. 5, pp. 385-392, 1998.

[24] Center for Disease Control and Prevention, "Missed opportunities for pneumococcal and influenza vaccination of Medicare pneumonia inpatients-12 Western states, 1995," Morbidity and Mortality Weekly Report, vol. 46, pp. 919-923, 1997.

[25] H. G. Bloom, D. A. Wheeler, and J. Linn, "A managed care organization's attempt to increase influenza and pneumococcal immunizations for older adults in an acute care setting," Journal of the American Geriatrics Society, vol. 47, no. 1, pp. 106-110, 1999.

[26] C. M. Healy, M. A. Rench, L. A. Castagnini, and C. J. Baker, "Pertussis immunization in a high-risk postpartum population," Vaccine, vol. 27, no. 41, pp. 5599-5602, 2009.

[27] Center for Disease Control and Prevention, "Use of standing orders programs to increase adult vaccination rates. Recommendations of the advisory committee on immunization 
practices," Morbidity and Mortality Weekly Report, vol. 49, no. 1, pp. 15-26, 2000.

[28] P. R. Dexter, S. M. Perkins, K. S. Maharry, K. Jones, and C. J. McDonald, "Inpatient computer-based standing orders vs physician reminders to increase influenza and pneumococcal vaccination rates: a randomized trial," The Journal of the American Medical Association, vol. 292, no. 19, pp. 2366-2371, 2004. 

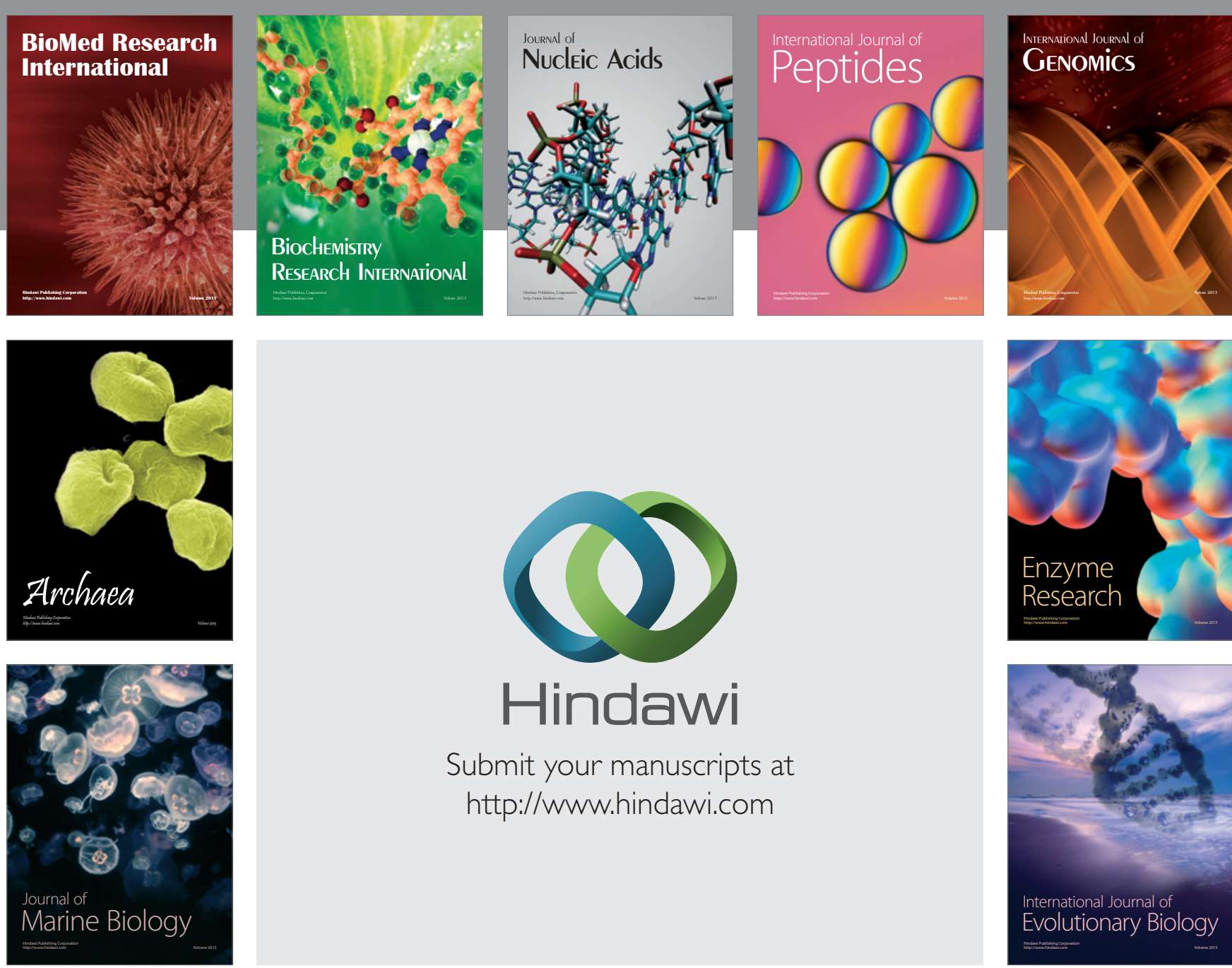

Submit your manuscripts at

http://www.hindawi.com
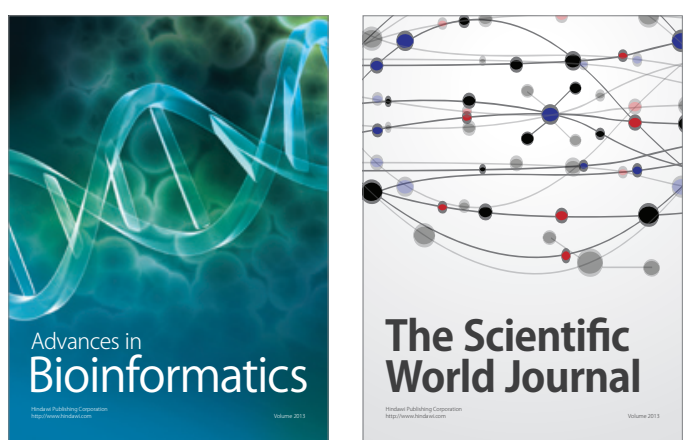

The Scientific World Journal

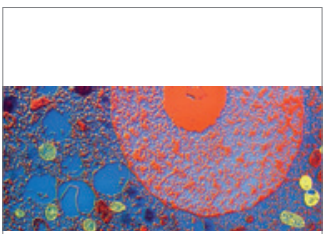

ISRN

Cell Biology

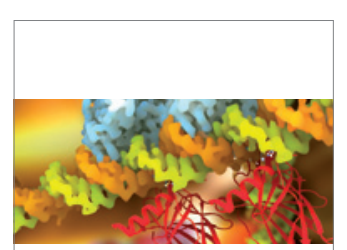

ISRN

Molecular Biology
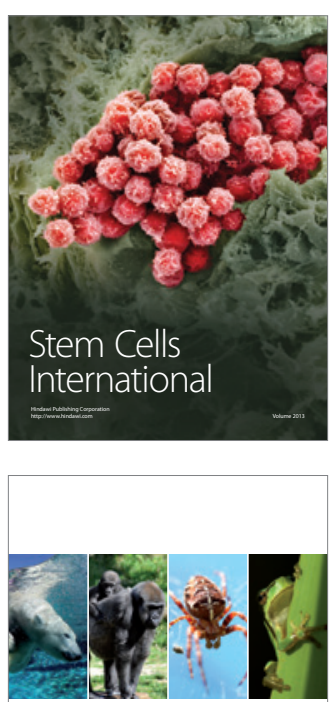

ISRN

Zoology

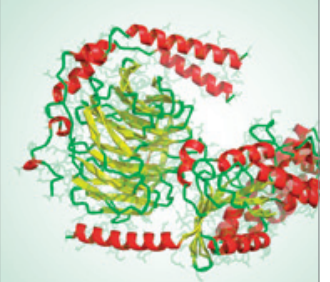

Signal Transduction

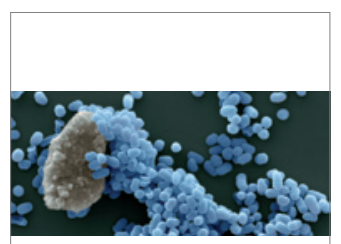

ISRN

Biotechnology
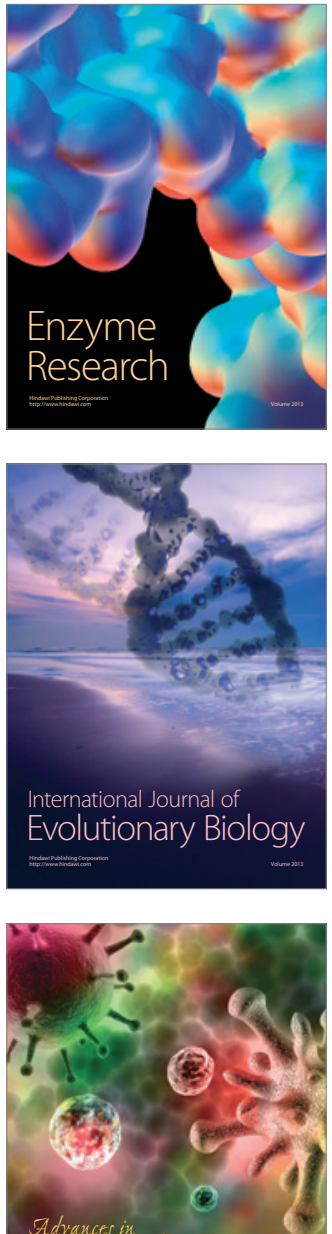

\section{Virology}

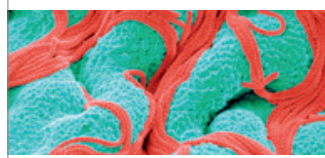

ISRN

Microbiology 